\title{
No Anterior Movement of Hyoid During Swallowing
}

National Cancer Institute

\section{Source}

National Cancer Institute. No Anterior Movement of Hyoid During Swallowing. NCI

Thesaurus. Code C127239.

A finding of no anterior movement of hyoid during swallowing. 